\title{
OWLGrEd: a UML Style Graphical Notation and Editor for OWL 2
}

\author{
Jānis Bārzdiņš, Guntis Bārzdiņš, Kārlis Čerāns, \\ Renārs Liepiņš, Artūrs Sproǵis \\ Institute of Mathematics and Computer Science, University of Latvia, \\ Raina blvd. 29, LV-1459, Riga, Latvia \\ Janis.Barzdins@lumii.lv, Guntis.Barzdins@lumii.lv, Karlis.Cerans@lumii.lv \\ Renars.Liepins@1umii.lv, Arturs.Sprogis@lumii.lv
}

\begin{abstract}
There have been several attempts to visualize OWL ontologies with UML style diagrams. Unlike ODM approach of defining a UML profile for OWL, we propose an extension to UML class diagrams (hard extension) that allows a more compact OWL visualization. The compactness is achieved through the native power of UML class diagrams extended with optional Manchester encoding for class expressions thus avoiding many explicit anonymous classes typical in ODM. We have implemented the proposed compact visualization in a UML style graphical editor for OWL 2. The editor contains a rich set of graphical layout algorithms for automatic ontology visualization, search facilities, graphical refactoring and interoperability with Protégé 4.
\end{abstract}

Keywords: OWL, graphical editor, visualization.

\section{$1 \quad$ Introduction}

OWL is gradually becoming the most widely used knowledge representation language that has been successfully deployed in a number of applications. Due to formal semantics and availability of reasoners for OWL, it is gaining popularity also in the software engineering community so far largely dominated by UML. Many newcomers have a background in software engineering where UML diagrams are the prevalent form of data modeling and they share many characteristics with OWL ontologies. Although the two languages are similar and it would be natural to reuse the existing familiarity, the UML notation cannot be used as is, because some OWL constructs have no equivalents in UML. A number of solutions have been proposed $[1,2,3]$, the most notable is ODM [1] that defines a UML profile for OWL. The main advantage of ODM approach is the possibility to use existing UML tools for ontology modeling. Meanwhile the price for this compatibility is more verbose notation that is hard to understand and does not facilitate comprehensibility.

In our opinion the most important feature for archiving readable graphical OWL notation is its maximum compactness. We are using UML notation as far as possible, but for concepts that are unique to OWL we extend UML with additional symbols and 
textual expressions (chapter 2). To make the notation usable in practice we have built an editor implementing it and supporting a number of features to ease ontology creation and exploration (chapter 3). The latest version of the editor can be downloaded from http://OWLGrEd.lumii.lv.

\section{$2 \quad$ Extended UML Notation for OWL}

The proposed graphical notation is based on UML class diagrams. For most features there is one to one mapping from OWL to UML concepts, e.g. ontologies to packages, OWL classes to UML classes, data properties to class attributes, object properties to associations, individuals to objects, etc. Meanwhile for OWL concepts not having a good UML equivalent, the following new extension notations were added:

- a field in classes for equivalent class, superclass and disjoint class expressions written in Manchester OWL syntax [4];

- a field in associations and attributes for specifying equivalent, disjoint and super properties as well as a field for specifying property characteristics, e.g., functional, transitive, etc.;

- anonymous classes containing equivalent class expression but no name;

- connectors for visualizing disjoint, equivalent, etc. axioms;

- boxes with connectors for n-ary disjoint, equivalent, etc. axioms;

- connectors for visualizing object property restrictions some, only, exactly, etc. The main advantage of these extensions is the option to specify class expressions in compact textual form rather than using separate graphical element for each logical class, constructor (and, or, not) and restriction, in cases where the expression is referenced only once. If the expression is referenced in multiple places, it can optionally be shown as an anonymous class. An anonymous class is also used as a base for property domain/range specification, if this domain/range is not a class.

To better understand the proposed notation lets consider an example in Figure 1 that depicts the popular African wildlife ontology [5] using our extended UML notation. At first glance it is very similar to an ordinary class diagram, therefore it should be immediately understandable by most people with software engineering background. On a closer inspection we can see that it also includes a number of extensions, namely classes Carnivore and Herbivore have a field that contains the equivalent class expression in Manchester syntax, there are a number of red connectors that correspond to property restrictions and there is a line between Plant and Animal for showing that they are disjoint. We believe that the example is intuitive and the meaning of the newly introduced symbols can be easily guessed, e.g. Tastyplant must be eaten by some Carnivore and some Herbivore. Super properties are depicted as a text next to subproperty's name, e.g. $\{<$ eaten-by\} next to subproperty name eaten-by-animal (symbol ' $<$ ' corresponds to 'subproperty' in UML notation).

We propose alternate notation possibilities for a number of OWL constructs, e.g. a graphical and textual form for subclass relation and for object property restrictions, and class disjointness specification by dependency links, by an n-ary disjointness symbol with multiple dependency links, or by a tag at a subclass fork symbol. 


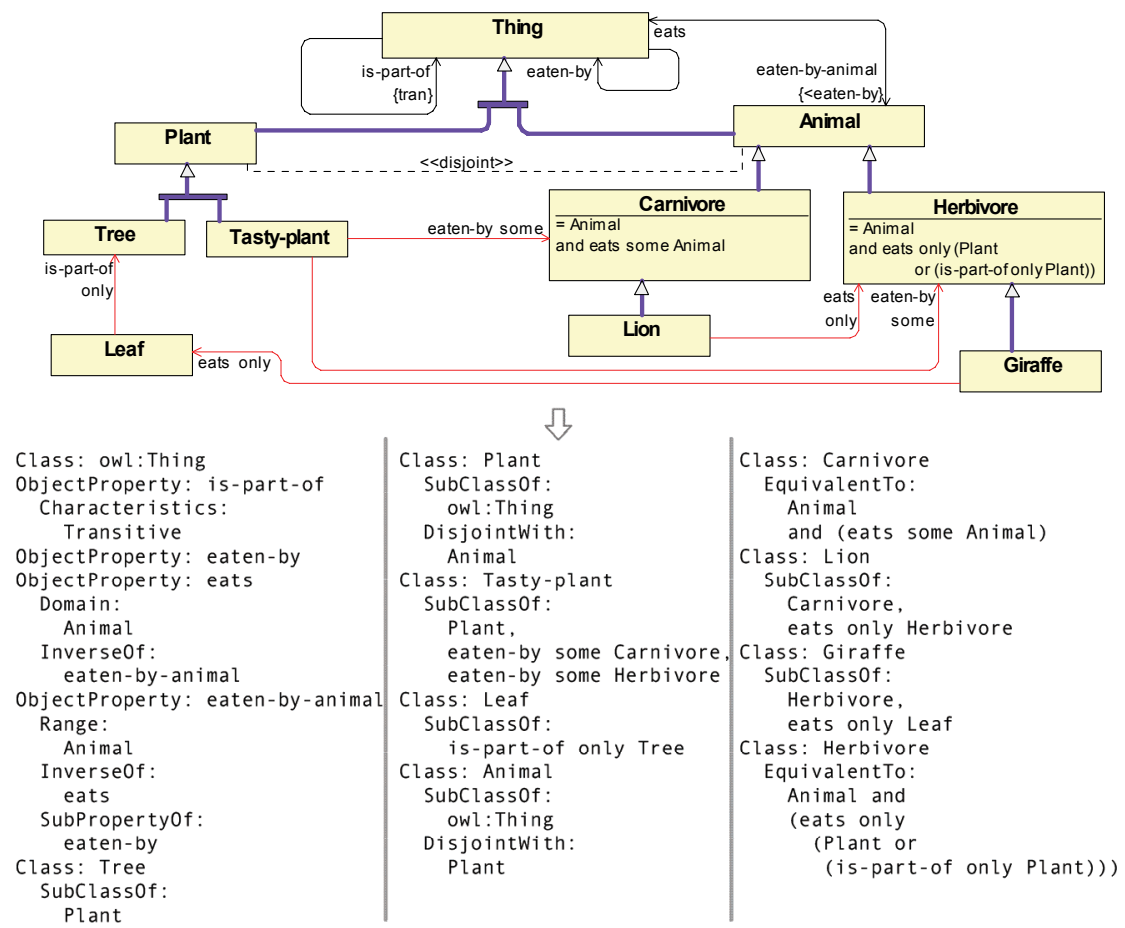

Fig. 1. Extended graphical notation and corresponding Manchester notation

\section{Services of the Editor}

The OWLGrEd editor is implemented using transformation driven architecture (TDA) $[6,7]$ and it has a number of special services to ease ontology development. One of the services is graphical refactoring that allows modifying graphical notation without changing semantics as long as the same concept can be expressed through different constructions. This feature allows the user to choose the most compact graphical format depending on the context and the taste. A typical situation illustrating the need for graphical refactoring is generalization and fork: if there is a single super class with multiple incoming generalization lines, a fork can be added to reduce multiple lines into a single line, and vice versa.

When ontologies become large, their management becomes difficult and besides support for import of modularized ontologies additional features are required from the editor. First, a good automatic layout is crucial for understanding large ontologies, therefore several alternative layout modes are supported. Second, searching for the specific element in large ontologies may become painful and irritating without an appropriate service. A search mechanism implemented in our editor allows finding the necessary element by specifying the value for one of its text fields. For example, it allows finding classes by their name or the value of any other text field. 
A more advanced service is full interoperability with Protégé 4 [8], an editor widely used by ontology developers. The interoperability allows ontology developers to use Protégé without changing their habits and afterwards visualize ontologies or their fragments in our graphical editor using various automatic layout algorithms along with manual layout tuning. Moreover, a user can specify the way ontologies will be visualized by selecting the exported information and notation options in preferences (e.g. only parts of ontology such as all classes and object properties but not instances can be sent for visualization).

In our graphical editor ontology developers can create new ontologies from scratch or alternatively edit graphically ontologies imported from Protégé; all graphically developed ontologies can afterwards be exported to Protégé from where they can be stored to various formats or checked with OWL reasoners.

\section{Conclusion and Future Work}

In this paper we described a new, compact OWL graphical notation and a betaversion implementation of the actual graphical editor. Our notation is based on UML class diagrams with additional constructs for OWL specific concepts - our aim is to cover full OWL 2.0 specification. The use of TDA platform [6, 7] has allowed developing OWLGrEd as a new editor that is not limited by possibilities offered by UML notation (including its profile mechanism), but where the extensions to the UML notation can be easily introduced, as necessary. The editor has also a number of features to ease ontology exploration and development, e.g. automatic layout algorithms and options for selecting which concepts shall be displayed. We are planning to add an option to store graphic layout information inside ontologies (we consider adding it as a special kind of annotations). We would also like to improve integration with Protégé, in particular, to synchronize ontologies in both tools after every editing step - current implementation allows exchanging only whole ontologies.

\section{References}

1. ODM UML profile for OWL, http://www.omg.org/spec/ODM/1.0/PDF/

2. TopBraid Composer, http://www.topquadrant.com/products/TB_Composer.html

3. Brockmans, S., Volz, R., Eberhart, A., Löffler, P. Visual Modeling of OWL DL Ontologies Using UML, Proc. of ISWC 2004, LNCS 3298, 2004, pp. 198-213.

4. OWL 2 Manchester Syntax, http://www.w3.org/TR/owl2-manchester-syntax/

5. Antoniou, G., van Harmelen, F. A Semantic Web Primer, 2nd Edition, MIT Press, 2008.

6. Barzdins, J., Rencis, E., Kozlovics, S. The Transformation-Driven Architecture, Proc. of 8th OOPSLA Workshop on Domain-Specific Modeling. Nashville, USA, 2008, pp.60-63.

7. Barzdins, J., Cerans, K., Kozlovics, S., Rencis, E., Zarins, A. A Graph Diagram Engine for the Transformation-Driven Architecture, Proc. of 4th MDDAUI. Florida, USA, 2009, pp.29-32.

8. Protégé, http://protege.stanford.edu/ 